\title{
Factores no tradicionales influyentes en la calidad de vida de los pacientes de hemodiálisis
}

\author{
Diana-Cristina Varela ${ }^{1}$, Mayra Guarín-Parra ${ }^{2}$, Natalia-Andrea Hincapié ${ }^{3}$, \\ Sandra-Patricia Hincapié ${ }^{3}$, Konniev Rodríguez ${ }^{4}$ \\ 1 Departamento de Investigación Clínica, Dialy-Ser, Bogotá, Colombia. \\ 2 Departamento de Estadística, Universidad Nacional de Colombia, Bogotá, Colombia \\ 3 Unidad de cuidados al final de la vida, Universidad San Buenaventura, Medellín, Colombia \\ 4 Departamento de Nefrología, Dialy-Ser, Bogotá, Colombia
}

\begin{abstract}
Resumen
Introducción: La prevalencia de pacientes con enfermedad renal crónica (ERC) en fases avanzadas, con requerimiento de diálisis, continúa creciendo. Una meta para el equipo tratante, el paciente y sus cuidadores es lograr un tratamiento que brinde un impacto en el pronóstico y en la calidad de vida (CV).

Metodología: En 100 pacientes con ERC en hemodiálisis, asignados en forma aleatoria, se evaluó la calidad de vida por medio del SF-36, se evaluó: capacidad funcional por medio del Karnofsky, capacidad de realizar las labores básicas de la vida diaria por medio del índice de Barthel, presencia de depresión por medio del test de Beck, funcionalidad familiar por medio del APGAR familiar, estado cognoscitivo por medio del Minimental y presencia de comorbilidades por medio del indice de Charlson correlacionado con la sumatoria de componentes físicos (PCS) y mentales (MCS) del SF-36, en pacientes pertenecientes a 2 unidades renales en Medellín y Bogotá, Colombia.

Resultados: Se encontró una disminución de la CV en los componentes físicos (PCS) y mental (MCS) respecto a la población general. Se encontró una correlación significativa entre PCS bajo y diabetes mellitus $(\mathrm{p}=0,008$ IC95\% 2,49-16). MCS bajo presentó relación con PTH ( $\mathrm{p}=0,046$ IC95\%: 0,000-0,014), el índice de Karnofsky ( $p=0,034$ IC95\% 0,244-5,984) y el test de depresión de Beck ( $p=0,000$ IC95\% $-13,606--6,253)$. Conclusiones: La CV de los pacientes en hemodiálisis se encuentra afectada con mayor impacto en PCS, existe relación significativa entre el MCS con estado funcional y depresión. Para mejorar la CV del paciente en hemodiálisis, se debe medir y hacer el seguimiento a variables no tradicionalmente medidas y optimizar el enfoque terapéutico dirigido a rehabilitación física, estado nutricional, funcional y psicológico.
\end{abstract}

Palabras clave: Enfermedad renal crónica, hemodiálisis, calidad de vida.

\section{Nontraditional factors influencing the quality of life of hemodialysis patients}

\begin{abstract}
Introduction: The prevalence of CKD patients in advanced stages with dialysis requirement continues to grow. A goal for the medical team, the patient and their caregivers is to provide a treatment that offers an impact on the prognosis and quality of life (QOL).

Methods: In 100 patients with CKD on hemodialysis, randomized assigned, the quality of life by SF-36 was assessed, it was evaluated the functional capacity using the Karnofsky, ability to perform basic tasks of daily life through the index Barthel, presence of depression using the Beck test, family functioning through family APGAR, cognitive state through Minimental and presence of comorbidities using the Charlson index correlated with the sum of physical components (PCS) and mental components (MCS) of the SF-36 in patients in two renal units in Medellín and Bogotá, Colombia.
\end{abstract}


Results: A decrease in QOL in the physical components (PCS) Mental components (MCS) regarding the general population. A significant correlation between low PCS and diabetes mellitus ( $-16 \mathrm{p}=0.00895 \% \mathrm{CI}$ 2.49) was found. Low MCS showed relation with PTH ( $\mathrm{p}=0.046$ 95\% CI 0.000-0.014), Karnofsky index $(\mathrm{p}=0.03495 \%$ CI 0.244 to 5.984) and Beck depression test ( $\mathrm{p}=0.00095 \% \mathrm{CI}-13.606$ to 6.253 ).

Conclusions: The QOL of hemodialysis patients is affected with more impact on PCS, there is significant relationship between MCS with functional status and depression. To improve QOL in hemodialysis patients, non traditionally-measured variables should be measured and monitored and the therapeutic focus should be optimized addressed to physical rehabilitation, nutritional, functional and psychological state.

Key words: Chronic kidney disease, hemodialysis, quality of life.

\section{Introducción}

S egún la Organización Mundial de la Salud (OMS), el 60\% del total de las muertes en el mundo es producido por enfermedades crónicas, el $80 \%$ de estas muertes sucede en países de ingresos bajos y medios entre los cuales figura $\mathrm{Co}-$ lombia.

El número de personas con enfermedad renal crónica (ERC) ha ido aumentando progresivamente en los últimos años. Los datos anuales USRDS 2013 reportan que 112.788 pacientes recibieron terapia de reemplazo renal (TRR), una tasa de incidencia ajustada de 357 por millón; la prevalencia de la terapia de diálisis fue de 395.656 personas durante el año $2011^{1}$. Es evidente que las tasas de enfermedad varían sustancialmente de acuerdo con la raza y la etnia. Colombia tiene una prevalencia de 621 pacientes por millón (ppm) y 22.300 pacientes en terapia de diálisis; y la tasa de incidencia fue de 81.7 ppm en $2012^{2}$.

Los estudios han demostrado que la ERC se asocia con aumento de la mortalidad y la morbilidad, sobre todo aumenta el riesgo para las enfermedades cardiovasculares ${ }^{3}$. La calidad de vida (QOL o $\mathrm{CV}$ ) es un indicador importante de la atención en salud, la experiencia del paciente y la valoración de la eficacia en diversas enfermedades crónicas. La evaluación de la calidad de vida se convierte en medida obligatoria en la evaluación de resultados y la eficacia del tratamiento en pacientes con diversas enfermedades, tales como enfermedad renal terminal ${ }^{4-6}$. En los pacientes con ERC, la calidad de vida no sólo proporciona la información importante en la vida diaria, sino también las percepciones del estado funcional ${ }^{7,8}$. La calidad de vida en los pacientes con ERC presenta repetidamente una puntuación baja en comparación con la de la población general ${ }^{9-11}$.

Entre los factores tradicionales conocidos influyentes en la calidad de vida de los pacientes de hemodiálisis encontramos factores demográficos como edad, género, nivel socioeconómico, religión, espiritualidad, la cercanía de la vivienda al sitio de diálisis y el apoyo familiar ${ }^{12,13}$; entre los factores clínicos encontramos: anemia ${ }^{14-17}$, hipoalbuminemia, uso de catéter, alteración de la paratohormona y del fósforo sérico ${ }^{18}$ y la presencia de comorbilidades tales como: diabetes, hipertensión arterial y depresión ${ }^{19-21}$.

\section{Sujetos y métodos}

\section{Pacientes}

En este estudio fueron incluidos 100 pacientes pertenecientes a 2 unidades renales ubicadas en Medellín y Bogotá, Colombia. Estos pacientes llevaban más de 3 meses en el programa de hemodiálisis en la institución. El consentimiento informado fue firmado luego de recibir información oral y escrita sobre el estudio, se contó con el aval del comité de ética de la institución.

En el estudio participaron 100 pacientes (41 mujeres y 59 hombres) mayores de 18 años. Se realizó un instrumento para la recolección de los datos, en el que se incluyeron aspectos sociodemográficos y 
clínicos como: edad, sexo, estado civil, nivel educativo, ocupación, acceso vascular, índice de masa corporal IMC y las comorbilidades; se obtuvieron datos de laboratorio como: hemoglobina, albúmina, potasio, calcio, fósforo, glucosa y hormona paratiroidea $\mathrm{PTH}$.

\section{Instrumentos}

La calidad de vida (CV) de los sujetos se evaluó mediante el Medical Outcome Study 36 Item Short-formsurvey (SF-36), el cual es un instrumento genérico de medida de la calidad de vida. Comprende 8 subescalas: función física, limitación por salud física, limitación por salud emocional, salud mental, funcionamiento social, dolor corporal, vitalidad y salud en general. Todas las subescalas están en un rango de 0 a 100, entre mayor valor, hay una mayor percepción de salud, el SF-36 está validado en español ${ }^{22-27}$. Además, hay medidas sumatorias del componente físico y mental (PCS y MCS).

La capacidad funcional de realizar labores cotidianas fue medida con el índice de Karnofsky. Las escalas de rendimiento de Karnofsky oscilan entre 0 y 100. Un puntaje más alto tiene mejor capacidad de realizar las actividades cotidianas. Se puede usar para determinar el pronóstico de un paciente, medir los cambios del paciente para funcionar; es un elemento predictor independiente de mortalidad ${ }^{28}$.

La capacidad de realizar las labores básicas de la vida diaria fue valorada por medio del índice de Barthel. La escala de Barthel es una escala de valoración funcional, valora la dependencia o independencia para 10 actividades de la vida diaria como son: comer, bañarse, vestirse, arreglarse, deposición, micción, ir al servicio, traslado sillón/cama, deambulación, escaleras y le asigna una puntuación de 0-5-10-15, en función del tiempo empleado para su realización y la necesidad de llevarlo a cabo obteniéndose una puntuación final de 0-100. La puntuación máxima de independencia es de 100 y la máxima de dependencia es de 0 ; los cambios se producen de 5 en 5 , no es una escala continua ${ }^{29}$.

Test de depresión de Beck es un cuestionario de 21 preguntas de respuesta múltiple, mide la severidad de la depresión, está compuesto por ítems relaciona- dos con síntomas depresivos, como la desesperanza e irritabilidad, cogniciones como culpa o sentimientos de estar siendo castigados, así como síntomas físicos relacionados con la depresión ${ }^{30}$.

El índice de comorbilidad de Charlson es un sistema de evaluación de la esperanza de vida a los 10 años, en dependencia de la edad en que se evalúa y de las comorbilidades del sujeto. Además de la edad, consta de 19 ítems, que si están presentes se ha comprobado que influyen de forma concreta en la esperanza de vida del sujeto. Inicialmente adaptado para evaluar la supervivencia al año, se adaptó, finalmente, en su forma definitiva para supervivencia a los 10 años. Se ha utilizado para otros muchos propósitos, entre ellos para el cálculo de costos a causa del padecimiento de alguna enfermedad crónica en enfermos de atención primaria ${ }^{31}$.

El Mini Mental State Examination (MMSE) de Folstein proporciona un análisis breve y estandarizado del estado mental. La versión de 35 puntos, explora 5 áreas cognitivas: orientación, fijación, concentración y cálculo, memoria y lenguaje ${ }^{32}$.

La funcionalidad familiar fue valorada por medio del APGAR familiar, el cual es un instrumento que muestra cómo perciben los miembros de la familia el nivel de funcionamiento de la unidad familiar de forma global, las puntuaciones sugieren una familia muy funcional, una familia moderadamente disfuncional o una familia con grave disfunción ${ }^{33,34}$.

Para garantizar unas condiciones estandarizadas, los cuestionarios fueron administrados durante la sesión de hemodiálisis y los participantes respondieron el cuestionario bajo la asistencia de una psicóloga entrenada. Todos los 100 sujetos cumplieron completamente los cuestionarios.

\section{Análisis estadístico}

El análisis estadístico se estructuró en 4 fases, iniciando con un análisis descriptivo univariado de las variables de interés observadas en el grupo de 100 pacientes, posterior a esto se realizó la prueba de normalidad de las variables continuas, con el fin de determinar la prueba de correlación a implementar de acuerdo con los supuestos que cumplía. 
Seguido a este proceso, se calculó la correlación de las variables continuas, si estas, en la prueba de normalidad, no tenían una distribución normal se implementó la prueba de correlación de Spearman, en el caso en que cumplieran con esta distribución, se utilizó la prueba de correlación de Pearson.

La realización de las pruebas de correlación permitió seleccionar las variables para el modelo de regresión lineal que se propuso al finalizar el análisis, si existían variables colineales o correlacionadas se seleccionaron las que aportaban más información al modelo y se excluyeron las demás, al finalizar el proceso, se planteó un modelo de regresión lineal múltiple que consideraba variables categóricas y continuas dentro de las regresoras, y que permitía calcular o estimar el valor de las variables de interés (PCS y MCS).

\section{Resultados}

Se analizaron 100 pacientes, 50 pertenecían a una unidad renal en Medellín y los otros 50 pertenecían a una unidad renal en Bogotá, Colombia.

La distribución por género fue de $41 \%$ femenino y $59 \%$ masculino, la edad promedio fue de $57 \pm 1,8$ años. Las variables clínicas se expresan en la tabla 1 .

En cuanto a la presencia de comorbilidades el 53\% de los pacientes se encontraba en hemodiálisis por nefropatía diabética y el $47 \%$ contaba con otra patología precursora del daño renal; el índice de Charlson mínimo para la población fue de $2(14 \%)$ por su condición de hemodiálisis, el puntaje máximo fue de 9 (1\%), el promedio del índice de Charlson en la población total fue de 4 (78\%).

La distribución por cada ítem de los test aplicados se encuentra expresada en la tabla 2.

La calidad de vida medida por el SF-36 mostró una disminución en todos los ítems con respecto a la población general, se encontró una disminución más marcada en la sumatoria de los componentes físicos con respecto a la sumatoria de los componentes mentales (Tabla 3).

\begin{tabular}{|l|l|l|}
\hline \multicolumn{3}{|c|}{ Tabla 1 } \\
\hline \multicolumn{3}{|c|}{ Características clínicas de la población } \\
\hline Variable clínica & Media & IC 95\% \\
\hline KTV & 1.96 & {$[1.39 ; 2.52]$} \\
\hline Hemoglobina & 11.61 & {$[11.20 ; 12.01]$} \\
\hline Albúmina & 5,41 & {$[2,93 ; 7,89]$} \\
\hline Potasio & 4.90 & {$[4.73 ; 5.06]$} \\
\hline Calcio & 8.71 & {$[8.51 ; 8.92]$} \\
\hline Fósforo & 5.39 & {$[4.99 ; 5.80]$} \\
\hline PTH & 610,55 & {$[507.1 ; 713.9]$} \\
\hline IMC & 24.29 & {$[23.37 ; 25.22]$} \\
\hline
\end{tabular}

En el modelo para PCS, la variable que mejor representación presenta para explicar el PCS bajo es padecer de diabetes mellitus ( $p=0,008$ IC95\% 2,4916), que explicó en $43,5 \%$ la PCS baja.

En el modelo para MCS, las variables que mejor explicaron un MCS bajo fueron la PTH ( $\mathrm{p}=0,046$ IC 95\%: 0,000-0,014), el índice de Karnofsky ( $p=0,034$ IC95\% $0,244-5,984)$ y el test de depresión de Beck $(\mathrm{p}=0,000$ IC 95\% -13,606- -6,253) lo cual indica que el modelo propuesto explicó en 64,6\% el puntaje bajo de MCS.

\section{Discusión}

Para la medicina del siglo XXI el enfoque tradicionalista de aliviar o mitigar la enfermedad debe no solo lograr el beneficio de mejorar el pronóstico, sino también, cumplir con mejorar la calidad de vida logrando así un tratamiento que proporcione años de vida útil para el paciente.

Los pacientes de hemodiálisis presentan en diferentes estudios una calidad de vida más baja que la población general ${ }^{19-11}$, la cual puede no mejorar significativamente después de iniciar la diálisis.

Los equipos interdisciplinarios a cargo de los pacientes con enfermedad renal crónica, deben abordar tempranamente los factores de riesgo conocidos 


\begin{tabular}{|c|c|}
\hline \multicolumn{2}{|l|}{ Tabla 2} \\
\hline \multicolumn{2}{|c|}{$\begin{array}{c}\text { Distribución de la población en los } \\
\text { test aplicados }\end{array}$} \\
\hline \multicolumn{2}{|l|}{ KARNOSFKY } \\
\hline 1. Inválido, en cama más del $50 \%$ & $1,0 \%$ \\
\hline 2. Gran atención, en cama menos del $50 \%$ & $8,0 \%$ \\
\hline 3. Requiere atención ocasional & $6,0 \%$ \\
\hline 4. Es capaz de cuidarse pero no de trabajar & $2,0 \%$ \\
\hline 5. Realiza actividades normales con esfuerzo & $6,0 \%$ \\
\hline 6. Actividades normales con síntomas leves & $24,0 \%$ \\
\hline 7. Normal & $53,0 \%$ \\
\hline \multicolumn{2}{|l|}{ BARTHEL } \\
\hline 1. Dependiente total & $3,0 \%$ \\
\hline 2. Dependiente grave & $1,0 \%$ \\
\hline 3. Dependiente moderado & $3,0 \%$ \\
\hline 4. Dependiente leve & $28,0 \%$ \\
\hline 5. Independiente & $65,0 \%$ \\
\hline \multicolumn{2}{|l|}{ BECK } \\
\hline 1. Normal & $67,0 \%$ \\
\hline 2. Leve perturbación & $17,0 \%$ \\
\hline 3. Depresión intermitente & $7,0 \%$ \\
\hline 4. Depresión moderada & $7,0 \%$ \\
\hline 5. Depresión grave & $2,0 \%$ \\
\hline \multicolumn{2}{|l|}{ MINIMENTAL } \\
\hline 1. Fase terminal & $2,0 \%$ \\
\hline 2. Demencia & $1,0 \%$ \\
\hline 3. Deterioro de la capacidad cognitiva & $10,0 \%$ \\
\hline 4. Sospecha de patología & $7,0 \%$ \\
\hline 5. Normal & $80,0 \%$ \\
\hline \multicolumn{2}{|l|}{ APGAR FAMILIAR } \\
\hline 1. Severamente disfuncional & $3,0 \%$ \\
\hline 2. Moderadamente funcional & $12,0 \%$ \\
\hline 3. Altamente funcional & $85,0 \%$ \\
\hline
\end{tabular}

\begin{tabular}{|l|l|}
\hline \multicolumn{2}{|c|}{ Tabla 3 } \\
\hline \multicolumn{2}{|c|}{ Puntajes del SF-36 por cada ítem y las sumatorias } \\
físicas y mentales \\
\hline Función física & $38,8 \pm 13,76$ \\
\hline Limitación por problemas físicos & $38,7 \pm 13$ \\
\hline Dolor corporal & $48,4 \pm 14$ \\
\hline Funcionamiento o rol social & $50,5 \pm 9,7$ \\
\hline Salud mental & $48,9 \pm 14,8$ \\
\hline Problemas emocionales & $46,36 \pm 13,8$ \\
\hline Vitalidad & $50,9 \pm 12,4$ \\
\hline Percepción salud en general & $45,9 \pm 10,5$ \\
\hline PCS & $38,3 \pm 12,2$ \\
\hline MCS & $53,4 \pm 13,7$ \\
\hline
\end{tabular}

como tradicionales, para brindar al paciente un adecuado estado nutricional, una construcción temprana de la fístula arteriovenosa, manejo adecuado de la anemia, entrenamiento y educación al paciente y a la familia, entre otros.

La calidad de vida fue analizada tanto en el componente físico como en el componente mental. El componente físico comprendido por las subescalas: función física, rol social, dolor corporal y limitación por problemas físicos, que conforman la sumatoria del componente físico (PCS), en este aspecto se encontró a la diabetes mellitus como el aspecto más importante que deteriora la calidad de vida de los pacientes que reciben hemodiálisis.

La diabetes mellitus es un precursor de daño macro y microvascular que conlleva no solo a la pérdida de la función renal si no también es causa importante de limitación visual, neuropatía y amputaciones, por lo cual la diabetes mellitus impacta negativamente en la calidad de vida de los pacientes e incrementa la mortalidad con respecto a la población de hemodiálisis ${ }^{19,35,36}$.

En cuanto al componente mental, las subescalas: vitalidad, percepción, salud mental y problemas emo- 
cionales conforman la sumatoria del componente mental (MCS). En este aspecto se encontró relación con la paratohormona, la limitación de la capacidad funcional de realizar labores cotidianas medida con el índice de Karnofsky y la presencia de depresión evaluada con el test de Beck, como los aspectos más relevantes que influyen en este componente.

La paratohormona PTH interviene en el metabolismo del calcio y fósforo, el trastorno de la regulación mineral ósea impacta negativamente en la calidad de vida, descrita previamente, en pacientes de hemodiálisis incidentes ${ }^{18}$ por mayor propensión a sufrir fracturas por enfermedad ósea y por mayor incidencia de eventos cardiovasculares asociados a calificación extraesquelética.

El índice de Karnofsky ha sido descrito como una escala que impacta en la mortalidad en la población de hemodiálisis, describiendo una mortalidad a un año del 68\% en los pacientes con índice de Karnofsky menor de $40^{36}$. Altos puntajes en esta escala han sido relacionados con una buena calidad de vida en hemodiálisis ${ }^{37,38}$.

El test de Beck es un instrumento creado para medir depresión. La presencia de depresión está descrita como un factor que impacta negativamente en la calidad de vida y ha sido referida hasta en el 41,7\% de los pacientes de hemodiálisis ${ }^{39}$. En este estudio se encontró una prevalencia del 33\%.

Para el modelo integrado de atención las decisiones clínicas deben enfocarse primariamente en las prioridades y preferencias del paciente individual, y el tratamiento se enfoca en influir sobre los factores modificables que le impiden al paciente gozar de salud y la sobrevida es solo uno de los objetivos de la atención ${ }^{40}$.
El tema de la calidad de vida del paciente en diálisis debe colocarse en un contexto de humanización, como un imperativo ético de la atención, para que se tomen decisiones pensando en su dignidad como ser humano y teniendo en cuenta su percepción de calidad de vida, en particular en aquellos pacientes con alta comorbilidad, bajo estatus funcional o con funciones cerebrales comprometidas.

Los equipos interdisciplinarios responsables del manejo de estos pacientes deben prestar mayor atención a variables no consideradas habitualmente en el plan terapéutico, pero no por eso menos reales, como la calidad de vida, condición emocional, dolor, sufrimiento, la carga del cuidador o los costos indirectos e intangibles ligados a la diálisis.

Tenemos el reto de ir más allá de unos indicadores clínicos biológicos y trascender hacia los demás componentes de la salud, como son el bienestar sicológico, espiritual y social del paciente. En otras palabras, hacer del cuidado del paciente en diálisis un arte con un componente moral de igual valor que el científico.

En conclusión, las escalas de funcionalidad y depresión deben ser incluidas como medidas rutinarias en los pacientes de hemodiálisis, como medida de la calidad de vida de esta población, es importante un abordaje multidisciplinario el cuál debe incluir la rehabilitación física, ya que es este aspecto el que presenta mayor impacto en la calidad de vida de este grupo y es la que menor intervención y manejo interdisciplinario tiene rutinariamente en las unidades renales.

\section{Conflicto de interés}

Los autores declaran no tener ningún conflicto de interés.

\section{Referencias bibliográficas}

1. U.S. Renal Data System, USRDS 2013 Annual Data Report: Atlas of Chronic Kidney Disease and End-Stage Renal Disease in the United States, National Institutes of Health, National Institute of Diabetes and Digestive and Kidney Diseases, Bethesda, MD, 2013.

2. Gómez RA. Renal disease in Colombia. Ren Fail. 2006;28(8):643-7. 
3. Weiner DE, Tighiouart $\mathrm{H}, \mathrm{Amin} \mathrm{MG}$ et al. Chronic kidney disease as a risk factor for cardiovascular disease and all-cause mortality: a pooled analysis of community-based studies. J Am SocNephrol 2004;15:1307-1315.

4. Kalantar-Zadeh K, Kopple JD, Block G et al. Association among SF36 quality of life measures and nutrition, hospitalization, and mortality in hemodialysis. J Am SocNephrol 2001;12:2797-2806.

5. Lopes AA, Bragg-Gresham JL, Satayathum S et al. Health-related quality of life and associated outcomes among hemodialysis patients of different ethnicities in the United States: the Dialysis Outcomes and Practice Patterns Study (DOPPS). Am J Kidney Dis 2003;41:605-615.

6. Lowrie EG, Curtin RB, LePain N et al. Medical outcomes study short form-36: a consistent and powerful predictor of morbidity and mortality in dialysis patients. Am J Kidney Dis 2003;41:1286-1292.

7. Unruh M, Benz R, Greene T et al. Effects of hemodialysis dose and membrane flux on health-related quality of life in the HEMO Study. Kidney Int 2004;66:355-366.

8. Kimmel PL, Emont SL, Newmann JM et al. ESRD patient quality of life: symptoms, spiritual beliefs, psychosocial factors, and ethnicity. Am J Kidney Dis 2003;42:713-721.

9. Kusek JW, Greene P, Wang SR et al. Cross-sectional study of healthrelated quality of life in African Americans with chronic renal insufficiency: the African American Study of Kidney Disease and Hypertension Trial. Am J Kidney Dis 2002;39:513-524.

10. Perlman RL, Finkelstein FO, Liu L et al. Quality of life in chronic kidney disease (CKD): a cross-sectional analysis in the Renal Research Institute-CKD study. Am J Kidney Dis 2005;45:658-666.

11. Tsai YC, Hung CC, Hwang SJ, et al. Quality of life predicts risks of end-stage renal disease and mortality in patients with chronic kidney disease. Nephrol Dial Transplant. 2010;25(5):1621-6.

12. Davison SN, Jhangri GS. The Relationship Between Spirituality, Psychosocial Adjustment to Illness, and Health-Related Quality of Life in Patients With Advanced Chronic Kidney Disease. J Pain Symptom Manage. 2012 Aug 20.

13. Davison SN, Jhangri GS. Existential and religious dimensions of spirituality and their relationship with health-related quality of life in chronic kidney disease. Clin JAm SocNephrol. 2010 Nov;5(11):1969-76.

14. Farag YM, Keithi-Reddy SR, Mittal BV, et al. Anemia, inflammation and health-related quality of life in chronic kidney disease patients. ClinNephrol. 2011 Jun;75(6):524-33.

15. Clement FM, Klarenbach S, Tonelli M, et al. The impact of selecting a high hemoglobin target level on health-related quality of life for patients with chronic kidney disease: a systematic review and meta-analysis. Arch Intern Med. 2009 Jun 22;169(12):1104-12.

16. Finkelstein FO, Story K, Firanek C, et al. Health-related quality of life and hemoglobin levels in chronic kidney disease patients. Clin J Am SocNephrol. 2009 Jan;4(1):33-8.

17. Covic A, Seica A, Gusbeth-Tatomir P, et al. Hemoglobin normalization trials in chronic kidney disease: what should we learn about quality of life as an end point? J Nephrol. 2008 Jul-Aug;21(4):478-84.

18. Johansen KL, Chertow GM. Chronic kidney disease mineral bone disorder and health-related quality of life among incident end-stage renal-disease patients. J Ren Nutr. 2007 Sep;17(5):305-13.

19. Mucsi I, Kovacs AZ, Molnar MZ, et al. Co-morbidity and quality of life in chronic kidney disease patients. J Nephrol. 2008 Mar-Apr;21Suppl 13:S84-91.

20. Soni RK, Porter AC, Lash JP, et al. Health-related quality of life in hypertension, chronic kidney disease, and coexistent chronic health conditions. Adv Chronic Kidney Dis. 2010 Jul;17(4):17-26.

21. Porter A, Fischer MJ, Brooks D, et al. Quality of life and psychosocial factors in African Americans with hypertensive chronic kidney disease. Transl Res. 2012 Jan;159(1):4-11.

22. McHorney CA, Ware JE Jr, Raczek AE. The MOS 36-Item Short-Form Health Survey (SF-36): II. Psychometric and clinical tests of validity in measuring physical and mental health construct. Med Care. 1993 Mar;31(3):247-63.

23. McHorney CA, Ware JE Jr, Lu JF, et al. The MOS 36-item Short Form Health Survey (SF-36): III. Tests of data quality, scaling assumptions, and reliability across diverse patient groups. Med Care. 1994 Jan;32(1):40-66. 
24. Vilagut G, Valderas JM, Ferrer M, et al. [Interpretation of SF-36 and SF-12 questionnaires in Spain: physical and mental components]. Med Clin (Barc). 2008 May 24;130(19):726-35.

25. Alonso J, Prieto L, Anto JM. [The Spanish version of the SF-36 Health Survey (the SF-36 health questionnaire): an instrument for measuring clinical results]. MedClin (Barc). 1995 May 27;104(20):771-6.

26. Alonso J, Regidor E, Barrio G, et al. [Population reference values of the Spanish version of the Health Questionnaire SF-36]. MedClin(Barc). 1998 Oct 10;111(11):410-6.

27. Ayuso-Mateos JL, Lasa L, Vázquez-Barquero JL. [Internal and external validity of the Spanish version of SF-36]. Med Clin (Barc). 1999 Jun 12;113(1):37.

28. Karnorfsky DA, Abelman WH, Craver LF, Burchenal JH. The use of nitrogen mustards in the palliative treatment of cancer. Cancer 1948;1:634-6456.

29. Mahoney F. Barthel D. "Functional evaluation: the Barthel Index". Md Med J 1965;14:61-65.

30. Craven, J.L., Rodin, G.M., Littlefield, C. “The Beck Depression Inventory as a screening device for major depression in renal dialysis patients". Int J Psychiatry Med 1988;18(4):365-374.

31. Charlson ME, Pompei P, Ales KL, et al. A new method of classifying prognostic comorbidity in longitudinal studies: development and validation. J Chron Dis, 1987;40(5):373-383.

32. Folstein, M., Folstein, S.E., McHugh, P.R. "Mini-Mental State" a Practical Method for Grading the Cognitive State of Patients for the Clinician. Journal of PsychiatricResearch, 1975;12(3);189-198.

33. Smilkstein G. The family APGAR: a proposal for a family function test and its use by physicians. J FamPract 1978;6:1231-9.

34. Bellon JA, Delgado A, Luna del Castillo JD, Lardelli P. Validez y fiabilidad del cuestionario de función familiar Apgar-familiar. Aten Primaria 1996;18(6):289-96.

35. Mandoorah QM, Shaheen FA, Mandoorah SM, et al. Impact of demographic and comorbid conditions on quality of life of hemodialysis patients: a cross-sectional study. Saudi J Kidney Dis Transpl. 2014 Mar;25(2):432-7.

36. Van Diepen M, Schroijen MA, Dekkers OM, et al. Predicting mortality in patients with diabetes starting dialysis. PLoS One. 2014 Mar;4;9(3):e89744.

37. Arogundade FA1, Zayed B, Daba M, Barsoum RS. Correlation between Karnofsky Performance Status Scale and Short-Form Health Survey in patients on maintenance hemodialysis. J Natl Med Assoc. 2004 Dec;96(12):1661-7.

38. Grincenkov FR1, Fernandes N, Chaoubah A. Factors associated with the quality of life of incident patients on PD in Brazil (BRAZPD).J Bras Nefrol. 2011 Mar;33(1):38-44.

39. Andrade CP, Cruz MC, Urrutia M, et al. Evaluation of depressive symptoms in patients with chronic renal failure. J Nephrol. 2010 Mar-Apr; 23(2):168-74.

40. Tinetti ME, Fried T. The end of the disease era. Am J Med. 2004 Feb 1;116(3):179-85. 